\title{
Toward More Brilliant Quantitative X-ray Analysis in (S)TEM
}

\author{
M. Watanabe
}

Dept of Materials Science and Engineering, Lehigh University, Bethlehem. PA, USA.

X-ray energy dispersive spectrometry (XEDS) has been used as a primary approach to gather elemental information from thin-film specimens in transmission electron microscopes (TEMs) because of its robust and relatively simple nature. In addition, it is very straightforward to identify most of elements immediately by acquiring an X-ray spectrum. Quantitative X-ray analysis can be performed by the conventional Cliff-Lorimer ratio method with so called Cliff-Lorimer k-factors [1], which are accessible in any modern XEDS systems. To improve quantitative X-ray analysis, the $\zeta$-factor method has been developed with built-in thickness determination for X-ray absorption correction. This approach was born initially in Kyushu University with Professors Nemoto and Horita [2] and significantly improved to the current form including various new feature with Professor Dave Williams in Lehigh [3,4]. In a thin-film specimen, the characteristic X-ray intensity from an element $\mathrm{A}, I_{\mathrm{A}}$, is simply proportional to the mass thickness $\rho t$ and the composition $C_{\mathrm{A}}$. and the $\zeta$-factor is defined to correlate those parameters as:

$$
\rho t=\zeta_{A} \frac{I_{A}}{C_{A} D_{e}}
$$

where $\zeta_{\mathrm{A}}$ is a proportionality factor to connect $I_{\mathrm{A}}$ to $\rho t$ and $C_{\mathrm{A}}$, and $D_{\mathrm{e}}$ is the total electron dose in the number of electrons during acquisition (the beam-current measurement is essential for this approach). The simultaneous determination of the composition and thickness provides further information including the analyzed volume with beam broadening, the number of atoms, or even the impurity enrichment at a grain boundary. Due to the built-in absorption correction, the $\zeta$-factor method is applicable to quantification of light elements such as oxygen $[4,5]$, and down to even carbon and boron [6]. Nowadays, the $\zeta$-factor method is available in the DTSA-II platform [7] or in some commercial XEDS systems from manufactures.

The determination of $\zeta$-factors is still one of the key challenges in this method. As shown in the equation above, the $\zeta$-factors can be determined from pure-element thin foils as long as the thickness is known. More efficiently, a series of the $\zeta$-factors for $\mathrm{K}$ lines can be determined from a single spectrum of the standard reference material 2063a glass thin-film issued by National Institute of Standard and Technology (NIST) [4]. This determination approach needs to be expanded to L and even M line families, which requires several standard specimens. A set of universal standards is under development for the $\zeta$-factor determination of whole X-ray lines. For example, a combination of NIST glasses K2437, K2580 and K3160 can provide wide energy ranges for X-ray lines: $\mathrm{K}$ lines of $\mathrm{O}-\mathrm{Zr}(0.5-17 \mathrm{keV})$, L lines of Fe-U (0.7-14 keV) and some of M lines (below $4 \mathrm{keV}$ ) (Fig. $1 \mathrm{a}-\mathrm{c})$. For $\zeta$-factor determination, the knowledge of specimen thickness is essential. However, by preparing the wedge-shape thin-specimens, the $\zeta$-factors can be determined without thickness information if multiple spectra are measured from different thickness regions [2]. These wedge-shape thin specimens can be prepared by focused ion-beam (FIB) instruments (Fig. 1d). Figure 2 shows $\zeta$-factors for $\mathrm{K}$ and $\mathrm{L}$ lines determined from the set of wedge-shaped NIST glass specimens. The estimation of K-line $\zeta$-factors is superimposed well on the measured values in the whole energy range up to $\sim 17 \mathrm{keV}$. For the L-line $\zeta$-factors, two different estimated series based on different cross-section models are also plotted as well as the measured values. There is a large discrepancy between 
the measure and estimated $\zeta$-factors for the $\mathrm{L}$ lines, especially at the higher energy range. Further models for estimation of the L line family are required. However, the $\zeta$-factor method promotes quantitative Xray analysis to the next level in combination with user friendly software and new standard specimens [8].

\section{References:}

[1] G. Cliff \& G.W. Lorimer, J. Microsc. 103 (1975), p. 203.

[2] M. Watanabe, Z. Horita and M. Nemoto, Ultramicrosc., 65 (1996), p. 187.

[3] M. Watanabe and D.B. Williams, Z. Metallk., 94 (2003), p. 307.

[4] M. Watanabe and D.B. Williams, J. Microsc., 221 (2006), p. 89.

[5] S. Fladischer and W. Grogger, Ultramicrosc., 136 (2014), p. 26.

[6] C. Marvel et al., Ultramicrosc., (in review).

[7] N.W.M. Ritchie, DTSA-II, available through https://cstl.nist.gov/div837/837.02/epq/dtsa2/.

[8] The author wishes to acknowledge Dr. Dale Newbury for providing NIST glass samples.
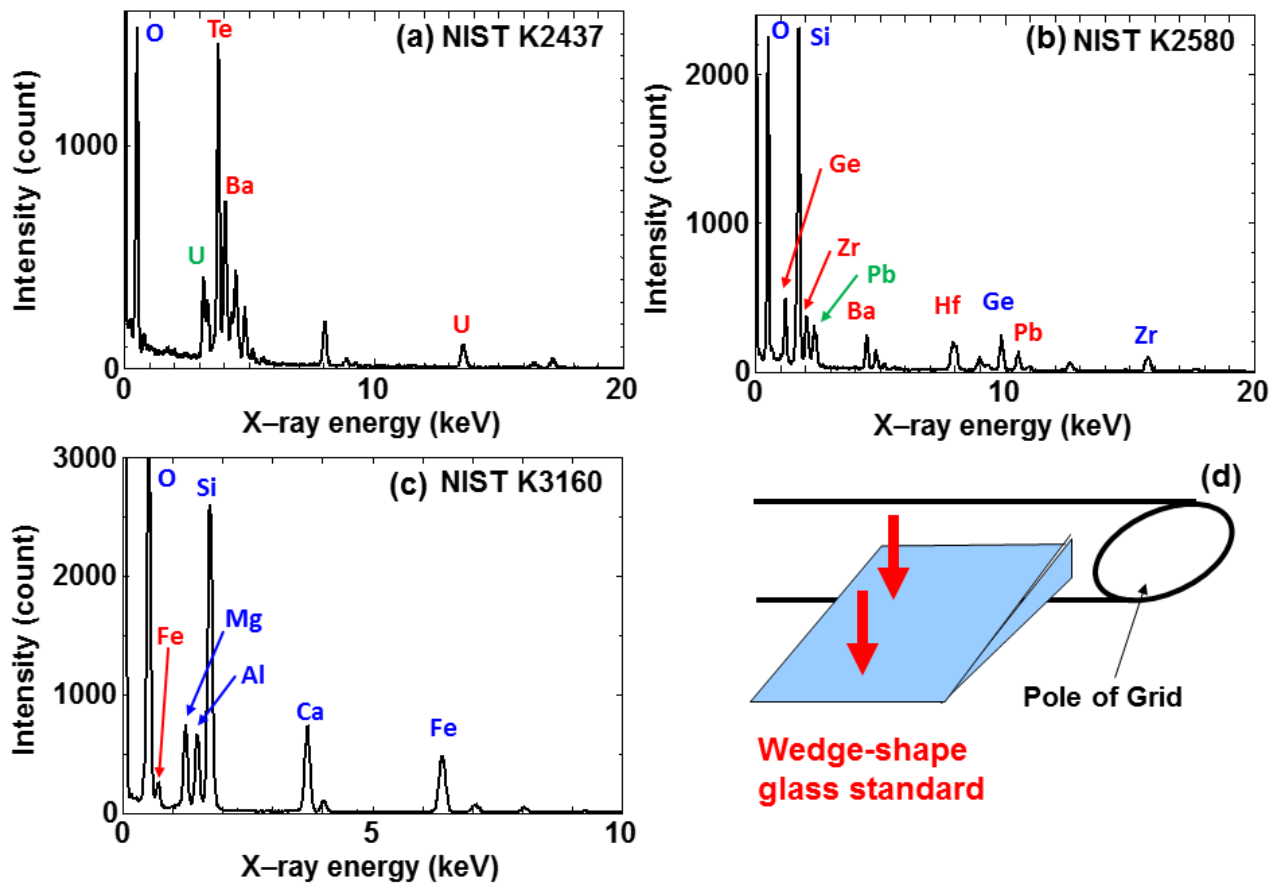

Figure 1. XEDS spectra from NIST glasses K2437 (a), K2580 (b) and K3160 (c). (d) A schematic diagram of an FIB-prepared wedge-shape specimen for $\zeta$-factor determination.
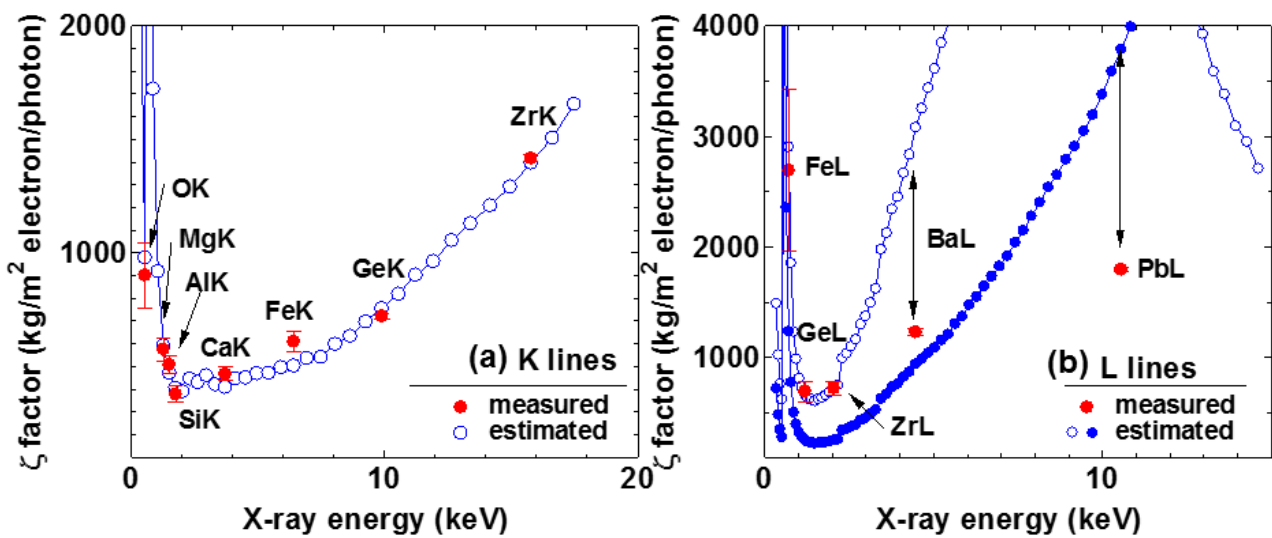

Figure 2. Determined $\zeta$-factors for K lines (a) and L lines (b) with estimated values. 